\title{
Das Vorkommen von Acetylcholin im Blut nach experimentellen Verbrennungen
}

\author{
Von Felix-Adolf Hoppe-Seyler $\dagger$ und Norbert Schümmelfeder* \\ Aus dem Physiologisch-chemischen Institut der Ernst-Moritz-Arndt-Universität \\ zu Greifswald
}

(Z. Naturforschg. 1, 696-699 [1946]; eingegangen am 26. Juli 1946)

\begin{abstract}
Ob sich im Blut nach lokalen Verbrennungen von Extremitäten, welche vorübergehend während dieser Schädigung aus dem Kreislauf ausgeschaltet waren, freies Acetylcholin $(\mathrm{ACh})$ findet, und ob das Auftreten von $\mathrm{ACh}$ bzw. acetylcholinwirksamer Stoffe von ursächlicher Bedeutung für das Zustandekommen des Schocks nach solchen Schädigungen sein kann, wurde in Versuchen geprüft. Acetylcholin-Bestimmungen führten wir an Hunden, bei welchen Hautverbrennungen an Extremitäten gesetzt wurden, in Pernocton-Chloralose-Narkose aus, unter gleichzeitiger Registrierung des Blutdrucks. Die Extremitäten wurden während der Schädigung durch Anlegen einer Esmarchschen Blutleere aus dem Kreislauf ausgeschaltet. Nach Lösen der Blutleere kam es zum Schock. Nach vorherigen Gaben von Atropin und Eserin wurde in eserinhaltiger Auffanglösung Blut aufgenommen und $\mathrm{ACh}$ im Vollblut bzw. Plasma ausgewertet. Weitere Blutproben wurden zur Auswertung nach dem von Kahls on entwickelten Verfahren enteiweißt und konzentriert. Alle Blut- bzw. Plasmaproben sowie die Konzentrate wurden am Blutegelpräparat nach Minz ausgewertet. Sofort nach Anlage der Blutleere waren nur bei einem von sieben Hunden nachweisbare Mengen von Acetylcholin im Blut vorhanden. Während der Verbrennung bei noch bestehender Abschnürung fanden sich im Blut bei drei von sieben Tieren nachweisbare Mengen von $\mathrm{ACh}$, in einem Fall bei noch unverändertem Blutdruck, bei zwei Tieren, während der Blutdruck bereits langsam abfiel. Nach Öffnung der Blutleere und Eintritt des Kollapses waren bei drei von sieben Tieren sicher nachweis- und auswertbare Mengen von ACh vorhanden, während bei zwei weiteren wahrscheinlich geringere, aber nicht mehr quantitativ auswertbare Mengen im Blut nachweisbar waren. Die gefundenen ACh-Mengen schwankten zwischen 1,25 und $15 \gamma / l$. Nach Einzelinjektionen und Dauerinfusionen von ACh-Lösungen wurden wesentlich größere ACh-Mengen im Blut gefunden (bis $60 \gamma / l$ ). Lang andauernde Infusionen mit Gaben bis zu $15 \mathrm{mg}$ ACh in der Minute führten nicht zum tödlichen Kollaps. Bei faradischer Reizung der Halsvagi fanden wir ACh im Blut größenordnungsmäßig etwa in der gleichen Menge wie bei den Schockversuchen. Die Blutdrucksenkungen waren jedoch nach den Vagusreizungen wesentlich geringer. Das Auftreten von ACh im Blut bei unseren Versuchen wird daher als Folgeerscheinung und nicht als Ursache der Kreislaufveränderungen angesehen.
\end{abstract}

$\mathrm{F}$ ast allgemein ist man der Ansicht, daß bei schweren Verbrennungen toxische Stoffe frei werden, die $\mathrm{zu}$ einem Kollaps führen können. Unter dem Gesichtspunkt, die Resorption derartiger Stoffe zu verhindern, wurde die Behandlung von Verbrennungen mit Gerbsäure (Tannin) eingeführt'. Auch die Abtragung des verbrannten Gewebes nach chirurgischen Grundsätzen² wird mit der Notwendigkeit, die Resorption toxischer Substanzen zu verhindern, begründet. Ein Beweis aber dafür, daß solche bei der Verbrennung frei werdende toxische Substanzen als Hauptursache für die Ausbildung kollaps- und schockartiger Kreislaufveränderungen in Betracht kommen, ist bisher nicht erbracht. Eine große Anzahl von Untersuchungen über die Rolle des Histamins bei der Verbrennung liegt vor. Ein Teil der Untersucher glaubt, dem Histamin eine maßgebliche Be-

* Patholog. Institut der Universität Münster i. W.

1 E. C. D a vid s on, Surgery, Gynecol. Obstetr. 41, 202 [1925]; W. C. Wils on, Med. Res. Council, ind. Health Des. Bvard, Rep., No. 141 [1929].

${ }_{2}$ P. Tschmarke, Dtsch. Z. Chir. 44, 346 [1897]. 
deutung zumessen $\mathrm{zu}$ müssen, während andere dies bestreiten ${ }^{3}$.

Bekannt ist, daß nach Öffnen einer Blutleere, die wegen einer Verletzung des entsprechenden Gliedes angelegt worden war, unter Umständen ein Kreislaufschock eintreten kann. Die Frage des Zustandekommens dieses Schocks ist ebenfalls ungeklärt. Bis vor nicht allzu langer Zeit wurde auch hierbei vor allem an eine Abgabe toxischer Stoffe aus dem verletzten Gewebe an das Blut gedacht. Im Tierversuch wurde aber festgestellt ${ }^{4}$, daß ein Schock auch dann eintritt, wenn die Esmarchsche Blutleere im Tierversuch an der unverletzten Extremität genügend lange angelegt und plötzlich geöffnet wird. Dabei unterstützt Erwärmung und verhindert Abkühlung der Extremität während der Blutleere den Eintritt des Kreislaufkollapses (Schwiegk u. Schöttler ${ }^{4}$ ).

Soweit uns bekannt, liegen keine Untersuchungen vor, ob bei Verbrennungen oder bei dem Schock nach Blutleere Acetylcholin im Blut auftritt. Wir stellten uns zur Aufgabe, diese Frage im Tierversuch bei Anwendung einer kombinierten experimentellen Schädigung, und zwar gleichzeitiger Blutieere und Verbrennung, zu untersuchen. Wir hielten es für vorteilhaft, hier eine solche kombinierte Schädigung anzuwenden, denn falls in dem geschädigten Gewebe eine Abgabe kreislaufwirksamer, „toxischer" Stoffe an das Blut erfolgt, welche an dem Zustandekommen des Kreislaufzusammenbruchs ursächlich beteiligt sind, ist wahrscheinlich mit einer gewissen Anhäufung derartiger Stoffe in der abgeschnürten Extremität und nach Lösen der Abschnürung mit einem plötzlichen Übertritt größerer Mengen in das zirkulierende Blut zu rechnen. Während der Auslösung des Schocks nach Abnahme der Esmarchschen Binde würde man also die besten

3 H. Kn o r r, Mitt. Grenzgebiete Med. Chir. 33, 326 [1921] ; H. Ma utner u. E. P. Pick, Münch. med. Wschr. 62, 1141 [1915]; dieselben, Biochem. Z. 127, 72 [1922]; dieselben, Naunyn-Schmiedebergs Arch. exp. Pathol. Pharmakol. 142, 271 [1929]; D. W. B en net u. N. D r u r y, J. Physiology 72, 288 [1931]; A. Si m onart, Arch. int. Pharmakodynam. Therap. 37, 269 [1930] ; G. S. B a r s o u m u. J. H. Ga d d u m, J. Physiology 85, $13 \mathrm{P}$ [1935]; dieselben, zit. n. J. H. G a d d u m, Gefäßerweiternde Stoffe der Gewebe, Thieme Leipzig [1936]; B. R o s e u. J. S. W. B r ow ne, Proc. Soc. exp. Biol. Med. 44, 182 [1940]; Loos, Arch. Dermatol. Syphilis 180, 50 [1940]; F. P. Underhill u. Kapsinow, J. Lab. clin. Med. 16, 823 [1931]; Harkins, Wilson u. Stuart, Proc. Soc. exp. Biol. Med. 32, 913 [1935] u. a.
Aussichten haben, etwaige Stoffe dieser Art zu fassen.

Unsere Versuche wurden an Hunden in PernoctonChloralose-Narkose durchgeführt. An diesen Tieren wurden möglichst hoch am Oberschenkel eine (Hund Nr. 1 und 2) bzw. beide (Tier Nr.3 bis 8) hinteren Extremitäten entsprechend dem Vorgehen von Schwiegk und Schöttler für 3 bis 4 Stdn. abgebunden. Während dieser Zeit setzten wir an den abgebundenen, geschorenen Extremitäten mittels einer Heizsonne bzw. Glühlampen Verbrennungen 1. bis 2. Grades. Die nicht abgebundenen Partien wurden sorgfältig gegen Wärmeeinwirkung abgedeckt. Zur Verhinderung des ACh-Abbaues im Blut erhielten die Tiere vor den jeweiligen Blutentnahmen Atropin und Eserin intravenös injiziert ${ }^{5}$. Die ACh-Bestimmungen erfolgten am mit Eserin sensibilisierten Blutegelmuskelpräparat ${ }^{\circ}$ in laufend während der einzelnen Versuche entnommenen Blutproben. Auswertungen erfolgten in Vollblut und Plasma sowie in Blutextrakten. Letztere wurden entspr. dem Vorgehen G. Kahlsons ${ }^{7}$ hergestellt durch Extraktion mittels Oxalsäure-Alkohol, Vakuumtrocknung, Aufnahme in eserinhaltiger Kaltblüter-Tyrode-Lösung und Einstellung mit Natriumbicarbonat auf $p_{\mathrm{H}} 7,5$. Da hierbei gelegentlich atypische Kontraktionen auftraten (Hypertonie? Kaliumwirkung ${ }^{8}$ ) wurde bei allen späteren Versuchen der Rückstand nach der Vakuumtrocknung mit absol. Alkohol aufgenommen, filtriert, nochmals getrocknet und dann erst in Tyrode-Lösung gelöst. Hiernach beobachteten wir keine atypischen Kontraktionen mehr. In Kontrollversuchen wurden dem Vollblut bzw. Plasma zugesetzte Mengen von ACh stets genau wiedergefunden. Nach Enteiweißung mit Oxalsäure-Alkohol fand sich zugesetztes $\mathrm{ACh}$ im Blutextrakt meist nur zu etwa $70 \%$, einmal unter absichtlich ungünstig gewählten Bedingungen nur zu $40 \%$ wieder. Die im Blutkonzentrat gefundenen Werte haben also nur qualitative Bedeutung, sie sind in jedem Fall zu niedrig. Die eigentliche Auswertung am-Rückenmuskel des Blutegels erfolgte mit geringen Modifikationen entsprechend der Technik der genauen Zeiteinteilung nach v. Muralt ${ }^{9}$ : Kontraktionsdauer 5 Min. (Stoppuhr), die Pausen zwischen den Auswertungen 25 Minuten.

4 Pa olucci, Arch. ital. Chir. 21, 4 [1928]; Panella, Ann. ital. Chir. 14, 1 [1935]; Wils on u. R o o me, Arch. Surgery 32, 334 [1936]; Allen, Arch. Surgery 38, 115 [1938]; H. Schwiegk u. W. H. A. Schöttler, Klin. Wschr. 22, 477 [1943].

5 W. Feldberg, Pflügers Arch. ges. Physiol. Menschen, Tiere 232, 88 [1933].

${ }^{6}$ B. M in z, Naunyn-Schmiedebergs Arch. exp. Pathol. Pharmakol. 167, 85 [1932]; 168, 292 [1932]; H. Fü hne r, Handb. biol. Arbeitsmeth. Bd. IV, 7 A, 421-609.

7 Naunyn-Schmiedebergs Arch. exp. Pathol. Pharmakol. 175, 189 [1934]; 175, 198 [1934].

8 Vergl. R. A m m o n, Klin. Wschr. 13, 1472 [1934].

9 Pflügers Arch. ges. Physiol. Menschen Tiere 245, 604 [1942]. 
Selbstverständlich ist mit der Möglichkeit $\mathrm{zu}$ rechnen, daß im tierischen Organismus Stoffe vorkommen können, welche wie ACh am eserinisierten Blutegelmuskel reagieren, und es ist klar, daß in allen Fällen, in denen ACh-Wirkung nachgewiesen und ausgewertet wird, genauer geprüft werden muß, ob es sich tatsächlich um diese Substanz handelt. Wie sich zeigte, war es für die vorliegende Fragestellung ohne Bedeutung, dieser Frage besonders nachzugehen. Immerhin haben wir uns in mehreren Fällen, in denen ACh-Wirksamkeit gefunden wurde, davon überzeugt, daß der Wirkstoff beim Stehenlassen in der Blutprobe rasch und im Konzentrat bei schwach alkalischer Reaktion langsamer völlig unwirksam wurde, sich in dieser Beziehung also wie $\mathrm{ACh}$ verhielt.

\section{Ergebnisse}

In Bestätigung der Befunde von A. Fleisch, I. Sibul und M. Kaelin ${ }^{10}$ fanden sich im Blut normaler Hunde in der Regel keine nachweisbaren Mengen von ACh. Nur bei einem (Hund Nr.3) unter sieben Versuchen fand sich aus unbekannten Gründen während der Blutleere in der ersten Blutprobe eine acetylcholinähnliche Wirksamkeit. Es handelte sich dabei nicht mit Sicherheit um $\mathrm{ACh}$, da die Kontraktionskurve des Blutegelmuskels atypisch war. In den anderen Fällen war auch im Blutkonzentrat zu dieser Zeit nichts nachzuweisen. Doch fand sich in den späteren Blutentnahmen noch vor Lösen der Blutleere bei drei Hunden ACh-Wirksamkeit. Bei einem kleinen Hund (Nr.2), dessen Blutdruck schon nach der ersten Blutentnahme von $20 \mathrm{ccm}$ um $20 \mathrm{~mm}$ Quecksilber absank und nach der Entnahme von weiteren $100 \mathrm{ccm}$ dauernd um $24 \mathrm{~mm} \mathrm{Hg}$ unter dem Ausgangswert blieb, war in der zweiten Entnahme eine ACh-Wirksamkeit von 1,25 bis $1,88 \gamma / l$ nachweisbar. Bei Hund Nr. 5 fanden wir in der zweiten Entnahme im Plasma eine ACh-Wirksamkeit, $(15,0 \gamma / l)$, ohne daß der Blutdruck wesentlich abgefallen war. Dagegen war bei Hund Nr. 6 der Blutdruck um $27 \mathrm{~mm} \mathrm{Hg}$ abgesunken, ehe $\mathrm{ACh}$ in der dritten Entnahme, und zwar im Blutkonzentrat in einer Konzentration von mehr als $1,25 \gamma / l$, nachweisbar wurde. Eine Zusammenfassung der Ergebnisse gibt folgende Tabelle. Ein Auswertebeispiel gibt die Abbildung.

Bei einem Hund (Nr.1) konnte, trotz zweimali-

10 Arch. int. Physiol. 44, 24 [1936].

\begin{tabular}{|c|c|c|c|c|}
\hline $\begin{array}{c}\text { Versuchs- } \\
\text { hund Nr. }\end{array}$ & $\begin{array}{c}\text {,Normal- } \\
\text { wert"** }\end{array}$ & $\begin{array}{c}\text { vor Lösung } \\
\text { der Blutleere }\end{array}$ & $\begin{array}{c}\text { nach Lösung } \\
\text { der Blutleere }\end{array}$ & $\begin{array}{c}\text { nach dem } \\
\text { Exitus }\end{array}$ \\
\hline & & & + & \\
1 & - & 0 & + & 0 \\
2 & - & + & $?$ & $?$ \\
3 & $?$ & $?$ & + & 0 \\
5 & 0 & + & 0 & 0 \\
6 & 0 & + & + & + \\
7 & 0 & 0 & 0 & 0 \\
8 & 0 & 0 & & \\
\hline
\end{tabular}

* Als „Normalwert" wurde die erste Blutentnahme be. zeichnet.

ger Blutleere und Verbrennung an einer hinteren Extremität von jeweils 4-stdg. Dauer, nach Lösen der Blutleere kein sicherer Schock erzeugt werden. Daraufhin wurde durch fortgesetzten Blutentzug ein Kreislaufversagen herbeigeführt. Hierbei trat im Blut eine $\mathrm{ACh}$ Wirksamkeit mit Sicherheit erst 1 Stde. nach Lösen der zweiten Blutleere auf, als der Blutdruck durch die vorhergegangenen Blutentnahmen bereits stark abgesunken war.

Es zeigte sich bei den
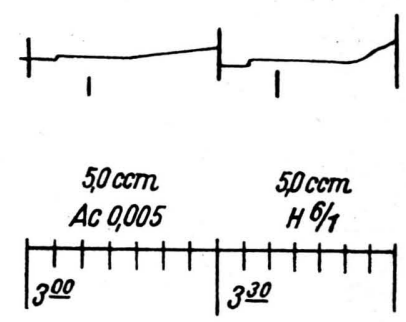

Ac $0,005=0,005 \gamma \mathrm{ACh}$ in 1 ccm Tyrode-Lösung. $H 6 / 1=$ Blutkonzentrat (nach Kahlson) einer während der Verbrennung und Blutleere entnommenen Blutprobe von Hund Nr. 6. übrigen 6 . Versuchen, daß kleine Mengen ACh bzw . acetycholinwirksamer Substanz zwar gelegentlich während der Versuche im Blut auftraten, daß jedoch die Konzentration dieser Stoffe bzw. ihre Wirkung nicht größer war als diejenige, welche Fleisch u. Mitarbeiter ${ }^{10}$, Blum u. Frane $\mathbf{l}^{\mathbf{1 1}}$ nach starken Blutverlusten oder erschöpfender Muskelarbeit fanden. Derartige Mengen können möglicherweise überhaupt bei stärkeren Schwankungen des Blutdrucks auftreten. Auch beim tödlichen Schock fehlte andererseits freies $\mathrm{ACh}$ in nachweisbaren Mengen im Blut bei manchen Versuchen vollkommen (vergl. Nr. 8). Diese Befunđe decken sich mit denen von I. Sibul ${ }^{12}$, der beim traumatischen Schock und nach Kreislaufversagen durch Entbluten ACh etwa in der gleichen Größenordnung

11 J.-D. Blum, Arch. int. Physiol. 46, 86 [1935]; W. Franel, Arch. int. Physiol. 41, 256 [1935].

12 Acta Comment. Univ. Tartuensis (Dorpatensis) A 34. Nr. 3 [1940]. 
fand. Auch die nach Adrenalininjektionen im Blut auftretenden und von $\mathrm{O}$. K r a y e r u. E. B. Verney ${ }^{\mathbf{1 3}}$ nachgewiesenen ACh-Mengen haben etwa die gleiche Höhe.

Selbstverständlich muß man mit der Möglichkeit rechnen, daß rasche und unter Umständen starke Schwankungen des ACh-Gehaltes im Blut bei Störungen der Kreislaufregulationen und namentlich der vegetativ-nervösen Regulationen vorkommen. Derartige, vorübergehende Steigerungen des ACh-Gehaltes zwischen den Entnahmen könnten dem Nachweis entgehen. Da jedoch schnelle Schwankungen für den verhältnismäßig protrahierten Verlauf des Kreislaufschocks nicht von ursächlicher Bedeutung sein können, wurden diese Fragen, welche von den hier zu erörternden abführen, nicht weiter verfolgt.

Mit Sicherheit kann man sagen, daß nach Verbrennungen jedenfalls nicht Acetylcholin als humoral wirksame „Depressorsubstanz“ von den geschädigten Geweben an das Blut abgegeben wird. Auch das Verhalten nach Atropingaben, nach denen der Blutdruck im Schock unvermindert abfiel, spricht hierfür. Unsere Versuche sagen andererseits natürlich nichts darüber aus, ob im Schock das Verhältnis Acetylcholin zu Cholinesterase an den Erfolgsorganen gestört ist.

$\mathrm{Um}$ anschaulich $\mathrm{zu}$ machen, daß die geringen Mengen an $\mathrm{ACh}$, welche bei einigen der Versuche gelegentlich gefunden wurden, ohne besondere Bedeutung für das Zustandekommen des Schocks sind, wurden einem Hund in Pernocton-Chloralose-Narkose steigende Mengen von ACh intravenös injiziert und schließlich infundiert. Gleichzeitig wurden ACh-Bestimmungen im Blut durch-

13 Klin. Wschr. 13, 1250 [1934]. geführt. Es konnten so z. B. nach einer Injektion von $600 \gamma \mathrm{ACh}$, die eine erhebliche, aber nur kurze Blutdrucksenkung verursachte, $60 \gamma \mathrm{ACh}$ im Liter Blut bei Auswertung im Vollblut nachgewiesen werden. Bei der Dauerinfusion konnten schließlich bis $15 \mathrm{mg}$. $\mathrm{CCh}$ pro Min. eingeführt werden, ohne einen tödlichen Schock hervorzurufen; während der Infusion von $500 \gamma \mathrm{ACh}$ je Min. wurden im Liter Blut $60 \gamma \mathrm{ACh}$ festgestellt. Die gefundenen ACh-Mengen sind bei diesem Versuch um ein Vielfaches höher als bei dem tödlichen Schock nach experimenteller Blutleere und Verbrennung. Um schließlich anschaulich zu machen, wie sich diese Werte denjenigen gegenüber verhalten, welche man nach Parasympathicusreizung und Eserinisierung erhalten kann, wurden bei einem Hund in Narkose beide Halsvagi faradisch gereizt und das Blut auf $\mathrm{ACh}$ untersucht. Nach wiederholter starker, aber nicht tödlicher Reizung fanden sich bis zu $20 \gamma / l$; nach tödlicher Reizung 20 bis $35 \gamma$ im Blut des rechten Herzens und $2,5 \gamma / l$ im Blut der Vena cava. Bei verhältnismäßig geringeren Blutdrucksenkungen als in den Schockversuchen treten hier also nicht wesentlich kleinere, sondern im Durchschnitt vielmehr höhere ACh-Mengen im Blut auf. Es zeigte sich im übrigen bei diesen Vagusreizversuchen deutlich, daß die Menge des im Blut nachweisbaren Acetylcholins in kein bestimmtes Verhältnis zur Blutdrucksenkung $\mathrm{zu}$ bringen ist.

Das gelegentliche Auftreten nachweisbarer Mengen von Acetylcholin im Blut möchten wir nicht als ursächliches Moment, vielmehr als Folgeerscheinung der starken Änderung der Kreislaufverhältnisse bzw. der vegetativ-nervösen Regulationen ansehen. 